\title{
Servicio comunitario del estudiante en el contexto de la educación superior venezolana
}

\author{
Ferrer, Juliana* \\ Clemenza, Caterina**
}

\section{Resumen}

El presente artículo analiza los alcances del Servicio Comunitario del Estudiante en el contexto de la Educación Superior venezolana. Basado en una investigación cualitativa, se realizó una entrevista de profundidad a los coordinadores de Servicios Comunitarios pertenecientes a cinco (5) Universidades públicas y privadas de la región Zuliana. Se evidencia por parte de la comunidad universitaria, diversas interpretaciones en relación al cumplimiento de la Ley, tal y como lo evidencian iniciativas descritas, refiriendo una resistencia implícita por parte del estudiante para el desarrollo de las iniciativas programadas. Se invita a reflexionar sobre la necesitad de fortalecer la acción social, de forma tal, que el estudiante pueda internalizar los valores de cooperación participación y responsabilidad, necesarios para lograr los objetivos de construir un ideal de ciudadanía, reconociendo que no puede darse en todos los contextos de la misma forma, pues depende de los procesos sociales en sus múltiples expresiones, de las coyunturas particulares existentes en cada comunidad y de la diversidad de relaciones entre actores sociales involucrados.

Palabras Clave: Servicio comunitario, responsabilidad participativa, ciudadanía social, Educación Superior.

Recibido: 23-07-08 • Aceptado: 07-03-09

* Postdoctorado en Gerencia de las Organizaciones. Postdoctorado en Ciencias de la Educación Doctora en Ciencias Gerenciales, adscrita al Instituto de Investigaciones de la Facultad de Ciencias Económicas y Sociales de la Universidad del Zulia.

E-mail: julianaferrer55@gmail.com

** Postdoctorado en Ciencias de la Educación Doctora en Ciencias Gerenciales, adscrita al Instituto de Investigaciones de la Facultad de Ciencias Económicas y Sociales de la Universidad del Zulia. E-mail: caterinaclemenza@yahoo.es 


\title{
Student Community Service in the Context of Venezuelan Higher Education
}

\begin{abstract}
This article analyzes the scope of Student Community Service in the context of Venezuelan higher education. Based on a qualitative investigation, in-depth interviews were carried out with Community Services coordinators belonging to five (5) public and private universities in the Zulia region. The university community evidences diverse interpretations related to fulfillment of the Law, as the described initiatives show, referring to an implicit resistance by the student to development of the programmed initiatives. The results invite reflection on the need to strengthen social action so the student can internalize the values of cooperation, participation and responsibility needed to achieve the objectives of building an ideal of citizenship, recognizing that this will not appear in all contexts in the same way, since it depends on social processes in their multiple expressions, on the particular circumstances existing in each community and on the diversity of relationships among the social actors involved.
\end{abstract}

Key words: Community service, participative responsibility, social citizenship, higher education.

\section{Introducción}

El compromiso social, de la labor educativa debe guiar la acción, con el fin de transformar las instituciones y comunidades verdaderamente democráticas, para lo cual éstas deben inmersas en su contexto inmediato y ser actores para el cambio desde una perspectiva de ciudadanía social, vinculado necesariamente a una perspectiva participativa inclusiva, como epicentro de una construcción de la convivencia comunitaria.

En definitiva, la colaboración activa de las instituciones universitarias, no es más que una preocupación ética para una educación de calidad, que exige el desarrollo de los valores desde la convivencia con las comunidades, cómo manera más coherente de darle significado al concepto de responsabilidad universitaria, bajo una cooperación que permita la armonización y cualificación de los contextos donde ésta se lleva a cabo. Este debe ser el fin último de una educa- ción que se defina como democrática, siendo necesario que todas y todos se planteen y den respuesta a las demandas comunitarias.

De acuerdo con lo anterior, el éxito de las políticas de participación y poder popular y particularmente la Ley de Servicio Comunitario para el estudiante de Educación Superior, depende de la posibilidad identidad colectiva, la cual permite la movilización de los miembros de la comunidad en búsqueda de soluciones conjuntas con la comunidad universitaria, permitiendo la posibilidad de enfrentar un estado de ineficiencia de recursos.

De allí la importancia del presente artículo, la corresponsabilidad entre el Estado- Universidad- Ciudadano, al permitir una valoración y la participación activa en la resolución de problemas de las comunidades donde el estudiante se inserta. El artículo está basado en una investigación cualitativa donde se recogen hallazgos significativos de los cursos de acción adelantados por cinco (5) Univer- 
Servicio comunitario del estudiante en el contexto de la educación superior venezolana Ferrer, Juliana y Clemenza, Caterina

sidades públicas y privadas de la región Zuliana a fin de analizar los alcances del servicio comunitario del estudiante en el contexto de la educación superior venezolana.

\section{La participación comunitaria en el marco de las tendencias sociales}

Las posiciones acerca de la participación comunitaria son diversas e, inclusive, contradictorias. Se pueden distinguir dos posiciones extremas la que apoya la inclusión de la participación en los programas y la que la rechaza. Se enfatiza sobre las consecuencias políticas y sociales y enfatizando las consecuencias de la participación comunitaria. El peso que cada criterio recibe en la argumentación y la forma en que ambos se articulan, permiten organizar las categorías de aceptación y rechazo de diversas concepciones (Bronfman y Gleizer, 1994).

De Roux, et al (1990) señalan que la participación comunitaria así definida, tiene implicaciones políticas que rebasan el marco de atención, por cuanto significa el ejercicio de poder y el fortalecimiento de la democracia, equivalente a un proceso de empoderamiento dentro de la vida social.

La postura de Winch, et al. (1991) es concebida desde la participación como un medio técnico para la implementación de programas. De esta manera plantean diferencias de enfoque que denominan desarrollo comunitario, en el que se privilegia el punto de vista de la población, equivalente a la postura que plantea a la participación como un fin en sí mismo.
Así concebida, la participación comunitaria deviene en una estrategia para maximizar la accesibilidad y disponibilidad de los servicios (Manderson et al, 1991). Esta concepción de la participación comunitaria como herramienta se piensa a sí misma como social y políticamente neutra. La participación es sólo un medio para llegar a un fin; el mejoramiento de la calidad de salud de la población.

Pero la participación nunca es absolutamente neutra, ni puede ser abstraída del contexto en el que tiene lugar; siempre tiene algún efecto sobre la estructura social de la comunidad, su organización y su capacidad de acción. Al concebir la participación como una estrategia sin consecuencias sociales, se corre el peligro de que ésta tenga efectos negativos sobre otros aspectos de la vida comunitaria (Gwatkin et al, 1979; Bronfman y Gleizer, 1994).

Refiere Bronfman y Gleizer (1994) como dentro de esta concepción se encuentra quienes interpretan a la participación comunitaria como una excusa para manipular política y socialmente a la comunidad. Su concepción coincide con la participación como medio político, pero difiere en el hecho de que en lugar de justificarla por su utilidad, cuestionan la inclusión de esta estrategia en los programas por los efectos negativos que tiene sobre la comunidad.

Lo paradójico de esta situación es que las interpretaciones surgen desde la misma corriente política; donde se argumenta a favor y en contra de la participación en función de los mismos objetivos de mejorar las condiciones de vida de los grupos menos favorecidos. De hecho, estas perspectivas aceptan, que las conse- 
cuencias políticas y sociales de la participación comunitaria no siempre están programadas y previstas inicialmente, ni están presentes de manera explícita. Entonces, la relación entre la participación comunitaria, sus consecuencias sociales y políticas y sus efectos, dependen de las formas que ésta adquiera en la práctica.

En otro orden de ideas, y trabajando la participación dentro de la concepción del fortalecimiento del capital social, surge ésta idea como parte interviniente en el desarrollo económico y social (Kliksberg, 2000), siendo un tema polémi$\mathrm{co}$, fácilmente susceptible de rápidos etiquetamientos ideológicos. Una de sus descalificaciones más frecuentes es considerarla como una iniciativa instrumental descontextualizada de la realidad. Sin embargo, se está produciendo cambios a nivel latinoamericano con apoyo de organismos internacionales quienes han adoptado la participación como estrategia de acción, que en diversos casos están institucionalizándola como política oficial.

Kliksberg (2000) refiere, como la participación comunitaria hoy se hace evidente; sobre todo, si se observa la basta brecha que separa en América Latina el discurso sobre la participación, de las realidades de implementación concreta; el consenso parece total, y la voluntad de llevar adelante la participación parece voluntaria.

Como todos los cambios significativos en la percepción de la realidad, surge la participación como una estrategia maestra de desarrollo, que posee anclajes profundos en necesidades emanadas de la realidad. De allí, la plataforma fundamental del nuevo interés surgido en torno de la participación comunitaria; ya que no utilizar los modelos participativos significará un costo de oportunidad en todos los aspectos organizativos planteados; pero además favorecerá la generación de costos directos innecesarios, que atentarán contra el cumplimiento de las metas y el desarrollo sustentable.

Bajo esta perspectiva la participación hará factibles condiciones para que la comunidad aprenda; se ejercite en el planeamiento y la gestión, y vea crecer sus capacidades; se fortalecerá entonces su posibilidad de sustentabilidad de cualquier programa participativo potenciando, la autoestima individual y colectiva; que se multiplicarán en energías y capacidades por la mejora de la calidad de vida futura.

\section{Servicio Comunitario como responsabilidad participativa en la ciudadanía social de futuro}

Cualquier análisis sobre reforma universitaria, implica el reconocimiento de una tarea compleja; sometida a fenómenos como la incertidumbre, la inestabilidad, la singularidad y los conflictos de valores. En la realidad cada institución aprende a sobrellevar factores contingentes e independientes a su control, que afectan el trabajo cotidiano. De allí que tal realidad, se convierta en un proceso de toma de conciencia para internalizar la responsabilidad de su actuación sobre los futuros egresados, las condiciones institucionales y sociales de la enseñanza y el compromiso ético de la misma, mientras adquieren los recursos necesarios para su formación integral. 
Servicio comunitario del estudiante en el contexto de la educación superior venezolana Ferrer, Juliana y Clemenza, Caterina

Hasta el momento, el proceso de enseñanza en las instituciones y particularmente en las universidades, se ha caracterizado por una marcada complejidad en las funciones básicas. Particularmente, Figueroa (2005) refiere, algunas características de la complejidad del trabajo docente, a la cual se enfrena cualquier iniciativa de cambio universitario entre ellas la multidimensionalidad y la imprevisibilidad. La primera referida a la cantidad de eventos y tareas diversas que debe cumplir el docente y la segunda subraya, la dimensión pública de la posición del docente ante lo inesperado de los eventos.

Frente a esta realidad, las instituciones de educación superior y particularmente las universidades, deben tener presente la contribución que de manera permanente aportan a un proceso educativo que tiene como punto de partida la formación de nuevos ciudadanos sociales, planetarios de futuro, teniendo como norte la tolerancia, solidaridad y desarrollo de los principios del respeto a los derechos humanos (Barylko, 2005); frente a una realidad marcada por la falta de identidad, la anomia y sobre todo el elevado anclaje con la racionalidad instrumental.

Hasta la fecha ha sido un error, según Morín $(1999,1995)$ considerar que la racionalidad (aun cuando ha contribuido al progreso de la humanidad), disponga de un espacio privilegiado; ya que está cimentada sobre un espacio frágil y puede quedar destruida por la propia condición humana.

A partir del reconocimiento de esa condición humana, se otorga una vital importancia al proceso educativo en el entendido, que éste está conciente de la ne- cesidad de desarrollar competencias genéricas, que vayan mas allá del ámbito del conocimiento y la racionalidad. Su formación debe rebasar el campo cognitivo para entrar en un proceso de carácter ético. Es decir, dada la complejidad de su práctica por la diversidad cultural y pluralidad de individuos y contextos, es recomendable que internalice principios y valores éticos institucionales que los apoyen para la generación de los líderes de futuro.

Para exponer la idea de las actuaciones éticas en instituciones educativas universitarias, es necesario tomar como referente los espacios donde se desenvuelve, las cuales siempre están enmarcadas en una actividad humana con una finalidad social; de modo que las actitudes necesarias para alcanzar su meta son actitudes morales; dichas actitudes hoy se modulan sobre el trasfondo de una ética cívica, para la cual tanto los miembros de cada organización como de una comunidad se convierten en interlocutores válidos para la acción (Ferrer, 2006).

Se hace preciso recordar que dichas instituciones se componen de personas, pero también en ellas existe un procedimiento aceptado, más o menos explícito a través del cual se toman las decisiones, de suerte que el responsable de las decisiones tomadas no es cada uno de los miembros de la organización, sino cada uno de los grupos de interés o protagonistas en su conjunto.

Así, su estructura, le dota de diversos rasgos, esenciales para considerarla como un agente moral; tales rasgos forman parte de su cultura institucional y la convierten en una organización humana. Etkin (1996) refiere como las cuestiones 
éticas en las organizaciones sociales no pueden tratarse como si fueran problemas de optimización de decisiones. La ética trata de resolver el nivel en que se ubica el problema, es una visión con los valores en juego, mandatos sociales a considerar en la situación y agrega deber ético, considerado un imperativo, no una obligación, sino una real y responsable frente a la realidad y dentro de los procesos históricos que la conforman.

En el mismo orden de ideas, Lozano (1999), considera la ética de las organizaciones, como una reflexión sobre el sistema, social, político y/o cultural, sobre las organizaciones y sobre sus actuaciones con los agentes sociales involucrados; el problema está en buscar cómo se articulan entre sí, bajo la llamada responsabilidad ética facilitando así, la participación activa de las comunidades en los procesos de desarrollo y de cambio estructural.

La responsabilidad ética de las organizaciones responderá así, a un hecho ya evidente en este nuevo contexto: La imposibilidad de separar lo económico, político, cultural y/o social. Atreverse a definir la responsabilidad ética de una organización, implica concebirla en un marco de actuación, respetando el pluralismo que caracteriza a las sociedades democráticas sin caer en el subjetivismo o relativismo (Llano, 1997), que impide determinar las verdaderas demandas sociales a las que ella debe responder y por tanto, a sus posibilidades de insertarse en espacios globales, a través una interacción, que le permita construir ciudadanía social de futuro, a través de la activa participación comunitaria, otorgándole a su vez legitimidad social.
De esta capacidad, aportación y cumplimiento de las expectativas que la sociedad ha depositado en cada protagonista social, deriva la responsabilidad y nace el balance social (Cortina et al, 1997), quién constituye la expresión de esa responsabilidad ética, puesto que ofrece información acerca del papel concreto que cada actor social cumple en la comunidad donde se desenvuelve.

\section{Los principios de acción participativa que albergan la iniciativa del servicio comunitario para el estudiante de Educación Superior}

En el nuevo contexto venezolano el Ministerio de Educación Superior, ha generado nuevas políticas y estrategias para el desarrollo del Sector en el periodo 2000-2010, la cual resume, entre otros aspectos, estructurar el sistema de educación superior; elevar la calidad académica de la instituciones, mejorar la equidad en el acceso y desempeño de los estudiantes, lograr una mayor pertinencia social de la instituciones y su interrelación con los distintos sectores de la sociedad y sobretodo, promover y fortalecer la participación comunitaria y el poder popular, a través de una cooperación permanente desde las instituciones de educación superior hacia las comunidades donde se insertan, con apoyo continuo de los espacios locales (Ministerio de Educación Superior, 2003).

Tal iniciativa, se suscribe en dos responsabilidades del gobierno nacional: primero, la transformación de la educación superior venezolana para dar respuesta al desarrollo nacional y la segunda, la partici- 
Servicio comunitario del estudiante en el contexto de la educación superior venezolana Ferrer, Juliana y Clemenza, Caterina

pación de la educación superior en la construcción de ciudadanos formados bajo condiciones de calidad, equidad social y cooperación, en la búsqueda de una participación activa, protagonista y corresponsales, de los cambios económicos, políticos y sociales que demanda una nueva configuración del aparato del Estado.

Se considera fundamental llevar a cabo la toma de decisiones, orientadas hacia la creación de comunidades autogestionadas con el objetivo de transformar el medio social. De la Riva (1993), describen la investigación participativa como una actividad integral que combina la investigación social, el trabajo educativo y la acción.

Entre sus características se destacan: El problema a estudiar se origina en la propia comunidad; el objetivo último de la investigación es la transformación estructural y la mejora de las vidas de los sujetos implicados; los beneficiarios son comunidades afectadas; la investigación participativa implica a la gente en su lugar de trabajo o su comunidad controla todo el proceso global del mercado; en la investigación participativa es central el papel que se asigna a fortalecer la toma de conciencia en la gente sobre sus propias debilidades, habilidades y recursos, así como su apoyo para movilizarse y organizarse; y el término investigador designa tanto a las personas del lugar de trabajo o a la comunidad, así como a aquellos que cuentan con un entrenamiento especializado (investigadores en el área). Es aquí donde los servicios comunitarios, logran ser espacio natural, contribuyendo a la generación de pro- yecto en función de las demandas jerarquizadas por las comunidades.

Tal y como puede visualizarse el sustento teórico tiene como soporte el papel activo del individuo, en el marco del contructivismo (Llano, 2006), evidenciado en la actuación que asumen las comunidades que participan en la propuesta problema, rompiendo de esta forma con la dicotomía separatista entre la teoría y la práctica. Este es un rasgo distintivo de la propuesta comunitaria, el cual conlleva a involucrar aquellas personas que están afectadas por los cambios planificados (Romero et al, 2006), las cuales tienen una responsabilidad primaria en cuanto a decidir acerca de la orientación de una acción críticamente informada que parece susceptible de conducir a una mejora y valorar los resultados de las estrategias sometidas a una verificación a través de una auditoría social.

Para dar viabilidad a tal política educativa, centrada en el servicio comunitario, se requiere de actores sociales que puedan construir intereses legítimos y aspiraciones, que en el campo educativo son necesarias para operativizar las misiones educativas (Plan Nacional de Desarrollo Económico y Social de la $\mathrm{Na}$ ción 2001-2007) que hoy, en el caso venezolano, tienen como finalidad insertar a un contingente importante de población al sistema formal existente y a su vez, formarlos integralmente, no sólo en términos de habilidades y conocimientos técnicos, si no en un cúmulo de competencias genéricas propias de la ética social; que garantice en éstos la inserción de un modelo participativo, capaces de generar un proceso transformador de la sociedad, de 
acuerdo a sus necesidades y demandas, adquiriendo un sentido de responsabilidad con respecto a su propio bienestar y contribuyendo de manera conciente, al proceso de desarrollo nacional.

Esta propuesta desde la perspectiva ética, se convierte hoy, en evidencia de este nuevo escenario social; quien bajo este cambio en el marco de actuación, reclama nuevas estrategias, nuevas formas de establecer su relación con la sociedad. En resumen, nuevas ideas para mantener y desarrollar credibilidad, teniendo como norte la responsabilidad social en la vinculación Universidad-Estado-Comunidades (Ferrer, 2006).

Ahora bien, teniendo a la responsabilidad como principio rector de acción, surge la Ley Orgánica de Participación Ciudadana y Poder Popular, aprobada en primera y segunda discusión el 22 de noviembre de 2001 y el 19 de julio de 2006 a los efectos de su disertación y opinión del pueblo venezolano.

La presente Ley tiene por objeto promover el desarrollo de políticas, instrumentos legales, sociales, así como mecanismos y normas relativas que contribuyan a garantizar; organizar e impulsar la participación ciudadana para el desarrollo del poder popular, participación expresada en forma directa, bien sea individual o colectiva, mediante la formulación de políticas públicas para darle consistencia social, material y cultural al hecho de la participación protagónica consagrada en la Constitución de la República Bolivariana de Venezuela (Ley Orgánica de Participación Ciudadana y Poder Popular, 2001).

Tal documento, interpreta como participación ciudadana, protagónica y corresponsable, la disposición consciente de las personas a involucrarse de manera colectiva o individual en la formulación, proyección, ejecución, control social y evaluación del Estado democrático y social de derecho y de justicia social. En este sentido, se reconoce la responsabilidad compartida de los integrantes de la comunidad y de las instituciones, en el proceso de formulación, ejecución, evaluación, seguimiento y control de las políticas públicas, para el beneficio común (Ferrer, 2006).

Bajo la realidad actual, se hace necesario orientar las incipientes iniciativas tomadas por las universidades venezolanas, como forma de avanzar en la solución de problemas demandados por las comunidades; a través de la detección de necesidades y construcción de proyectos sociales de pertinencia social, un conocimiento de sus demandas, convirtiéndolas en protagonista del proceso antes descrito; como forma de garantizar una autogestión donde sujeto y objeto se superponen e interrelacionan a favor del colectivo (Morín, 1999). Y es a partir de tal interrelación, donde se establecen vínculos de cooperación sujeto - sujeto, a fin de garantizar la determinación real de las necesidades sentidas por los afectados; como condición necesaria para que ellos sean principales protagonistas del proceso, dentro de las comunidades afectadas en una zona de influencia.

En el mismo orden de ideas surge la Ley de Servicio Comunitario, la cual está orientada a potenciar el trabajo comunitario que deben realizar los estudiantes en las comunidades. Su objetivo principal es que el estudiante que aspira un titulo universitario tenga la suficiente 
Servicio comunitario del estudiante en el contexto de la educación superior venezolana Ferrer, Juliana y Clemenza, Caterina

preparación académica y social, para no desligarse de su comunidad, que genere sentido de pertinencia en su entorno, de forma tal que al ser profesionales trabajen al servicio de las comunidades.

Igualmente, con esta Ley; se busca transformar las prácticas de las pasantías, dejando de un lado el individualismo, y profundizando en la importancia que tienen las mismas para la formación integral del individuo, de esta manera el estudiante deberá aportar sus conocimientos y servicios encaminados al desarrollo y mejora de las comunidades.

Dicha Ley, entro en vigencia a partir del 14 de Septiembre del 2006. A tales efectos se entiende como Servicio Comunitario, la actividad que deben desarrollar en las Comunidades los estudiantes de Educación Superior que cursen estudios de formación profesional, aplicando los conocimientos científicos, técnicos culturales, deportivos y humanísticos adquiridos durante su formación académica, en beneficio de la comunidad, para cooperar con su participación al cumplimiento de los fines del bienestar social, de acuerdo con lo establecido en la Constitución de la República Bolivariana de Venezuela y esta Ley (Asamble Nacional, LSC, art. 4). Igualmente, se entiende como "Comunidad", el ámbito social de alcance nacional, estadal o municipal, donde se proyecta la actuación de las instituciones de Educación Superior para la prestación del Servicio Comunitario.

Según lo establecido en el Artículo 2 de la Ley de Servicio Comunitario para el estudiante de Educación Supe- rior, se rige por los principios constitucionales propios de una cultura comunitaria: Solidaridad; Responsabilidad Social; Igualdad; Cooperación; Participación Ciudadana; Asistencia Humanitaria; Alteridad; entre los valores descritos explícitamente.

Así mismo, el artículo 7 de la Ley de Servicio Comunitario, establece los fines que se plantean para dicho servicio, destacando: la necesidad de fomentar en el estudiante, la solidaridad y el compromiso con la comunidad como norma ética y ciudadana; hacer un acto de reciprocidad con la sociedad; enriquecer la actividad de educación superior, a través del aprendizaje del servicio, con la aplicación de los conocimientos adquiridos durante la formación académica, artística, cultural y deportiva; integrar las instituciones de educación superior con la comunidad, para contribuir al desarrollo de la Sociedad Venezolana; y sobre todo, formar a través del aprendizaje del servicio, el capital social en el país.

En este sentido, se trata de proyectos de iniciativa de la propia institución de educación superior, sean elaborados de acuerdo al perfil académico de cada disciplina y a las necesidades de las comunidades. Además, dichos proyectos deben ofrecer soluciones metodológicas a los problemas planeados; a partir de la identificación de las necesidades y en la solución de problemas planteados por las comunidades, siempre tomando en consideración los planes de desarrollo nacional, estadales y municipales. 


\section{Algunas evidencias sobre la actuación de las Universidades respecto al Servicio Comunitario Estudiantil}

Las evidencias presentadas son producto de la aplicación de una entrevista a profundidad dirigida a los coordinadores de departamento de servicio comunitario de cinco (5) universidades públicas y privadas de los municipios Maracaibo y Jesús Enrique Losada del estado Zulia; específicamente la Universidad del Zulia (LUZ), la Universidad Bolivariana de Venezuela (sede Maracaibo) (UBV); la Universidad Nacional Experimental de la Fuerza Armada Bolivarianas (Núcleo Zulia) (UNEFAB); la Universidad Rafael Urdaneta (URU) y la Universidad Dr. Rafael Belloso Chacín (URBE):

Sus argumentos apuntan a destacar las herramientas puestas en práctica para llevar a cabo el servicio comunitario y satisfacer los requerimientos de las comunidades, según lo exigido por la Ley de Servicio Comunitario para el Estudiante de Educación Superior.

Se pudo evidenciar como existe una marcada expectativa en cuanto a la aplicación de la Ley de Servicio Comunitario, tanto por los involucrados en su coordinación, como por la comunidad estudiantil, lo que facilita el desarrollo de su puesta en marcha. En lo referente a las leyes que rigen el servicio comunitario, los coordinadores han liderado talleres para sensibilizar la comunidad universitaria en relación a los beneficios que dicha Ley otorga al cumplimiento de las funciones básicas universitarias y particularmente a la investigación y extensión, de- sarrollando proyectos de pertinencia social, que determinan el cumplimiento de la responsabilidad social universitaria.

De igual forma, en lo relativo a las actividades ofertadas, los coordinadores de las universidades, prestarán entre otros servicios, asesoría jurídica, comercial, de computación, cooperativas y educación, además crearán brigadas ambientalistas y proyectos de reciclaje y de rescate del ambiente; así como formación para voceros y voceras para la participación en los Consejos Comunales; esto como parte de las expectativas de las comunidades que se encuentran en las parroquias aledañas a cada una de las universidades.

Además, las universidades están dispuestas a ofrecer según sus áreas de competencia, otros servicios de acuerdo a la necesidad manifestada por las comunidades; lo cual implica formar un estudiante para que aporten sus conocimientos y servicios, encaminados al desarrollo y mejora de sus comunidades, permitiendo al mismo tiempo la integración de la institución de educación superior con la comunidad y contribuyendo así, al desarrollo de la sociedad venezolana.

Se evidenció además, por referencia de los coordinadores como ha existido en un primer momento una resistencia marcada al desarrollo de esta actividad por parte del estudiante; actitud que ha sido modificada luego de realizada la actividad, manifestando como dicho estudiante respondió finalmente con la actividad asignada y en su mayoría están dispuesto a continuar con la actividad comunitaria. Esto a excepción de la UBV y la UNEFA cuyos estudiantes realizan acción social desde su ingreso a la institu- 
Servicio comunitario del estudiante en el contexto de la educación superior venezolana Ferrer, Juliana y Clemenza, Caterina

ción y ya desarrollan de manera voluntaria actividades en las comunidades donde se insertan. Por lo que deben seguirse desarrollando y estableciendo estrategias basadas en el cambio social voluntario, que tenga por objeto la modificación de comportamientos para mejorar la situación de la población en su conjunto. De esta manera los estudiantes de educación superior podrán aplicar sus conocimientos científicos, técnicos, culturales, deportivos y humanísticos adquiridos durante su formación académica en la aplicación de proyectos comunitarios específicos, en beneficio de la comunidad, cooperando con su participación en el cumplimiento de los fines del bienestar social establecidos en la Constitución de la República Bolivariana de Venezuela y en las diversas leyes.

A continuación se presentan las iniciativas particulares manifestadas por las instituciones objeto de estudio.

\section{Universidad Bolivariana de Venezuela- Núcleo Zulia (UBV-Zulia)}

- Desde sus inicios viene desarrollando actividades orientadas al servicio comunitario, por las exigencias curriculares de los Programas Ofertados.

- En cada uno de los programas que esta institución ofrece, los estudiantes han trabajo de manera activa con las comunidades.

- Los estudiantes evalúan las problemáticas de las comunidades, esto con la finalidad de que adquieran pertinencia y ofrezcan soluciones en las situaciones acontecidas en su localidad.
- Los estudiantes realizan estudios de las necesidades presentes en los sectores, para conocer las comunidades que se encuentran en situación crítica, marginalidad, pobreza extrema, analfabetismo, entre otros. También desarrollan actividades extracurriculares, charlas ideológicas, intercambios deportivos, entre otros.

- Los estudiantes realizan campañas de concienciación (conciencia y sensibilización) sobre el ambiente. Además, realizan estudios del grado de contaminación por localidad.

- Los participantes del Programa de Estudios Jurídicos, actúan como intermediarios entre los jueces de paz y la comunidad para ayudar a la comunidad a resolver los problemas.

- Se está planificando un proceso de reuniones de equipos multidisciplinario, donde los estudiantes tengan participación para la creación del Reglamento Interno de la Universidad.

\section{Universidad Nacional Experimental de la Fuerza Armada Bolivariana-Núcleo Zulia (UNEFAB-Zulia)}

- Todavía no ha llegado el tiempo exigido para que los estudiantes cumplan con las actividades y proyectos de Servicio Comunitario.

- Se están planificando discusiones de la ley y la realización de foros donde los estudiantes tengan la posibilidad de conocerla, de estudiar sus deberes, objetivos y beneficios que ésta le ofrece, además de concientizarlos de la importancia de la misma. 
- Sin embargo, los estudiantes evalúan de manera permanente la problemática de sus comunidades, esto con la finalidad de que adquieran conciencia social y ofrezcan soluciones en las situaciones acontecidas en su localidad.

\section{Universidad del Zulia (LUZ)}

- Se están llevando a cabo reuniones para definir las diferentes modalidades que asumirá en práctica cada facultad en el cumplimiento de la Ley de Servicio Comunitario.

- Existe la posibilidad de crear una Dirección o Coordinación independiente que regule las actividades establecidas en la LSC.

- Se están revisando los planes de estudios de todas las carreras y realizando una preselección de las unidades curriculares con las que se podría cubrir la capacitación al estudiante y la aplicación práctica del desarrollo de Proyectos Comunitarios.

- Se esta estudiando la incorporación de una materia a nivel de todas las carreras, enfocada a la problemática económica y social venezolana.

- Se creará la unidad curricular Taller de Servicio Comunitario en todas las carreras.

- Se está estudiando incluir como obligatorio en las carreras Sociales y Humanisticas, una materia relacionada a los Consejos Locales de Planificación Pública.

- Se plantea una reforma curricular orientada hacia la formación integral del alumno y capacitarlo para ser útil en la sociedad, buscando propiciar la cohesión de los grupos sociales de una estructura global de naturaleza bio - psicosocial; cultivar valores de identidad, solidaridad, humanización y promover la realización de todas aquellas expectativas individuales y comunitarias demandadas desde el entorno universitario.

\section{Universidad Rafael Urdaneta (URU)}

- Se ha formulado el Reglamento Interno para llevar a cabo el Servicio Comunitario, en el cual se establece que los estudiantes deberán cumplir con actividades comunitarias aprobadas por los organismos respectivos, como el Ministerio de Educación y las autoridades responsables en cada área.

- Se comenzó a partir de enero 2007 a llevar a cabo el servicio comunitario para los estudiantes del $7 \mathrm{mp}$, $8 \mathrm{vo}$ y 9 no semestre de cada carrera.

- Se están llevando a cabo reuniones con el Ministerio de Educación, las Escuelas Zulianas de Avanzada de la Gobernación del Estado Zulia y la Alcaldía de Maracaibo para establecer los posibles proyectos a desarrollar.

- Con el Ministerio de Educación y las Escuelas Zulianas de Avanzada se estableció un convenio para que 200 estudiantes del área de Educación presten Servicio Comunitario en las escuelas respectivas.

- Los estudiantes de la carrera de Psicología aplicarán el servicio comunitario en el área de problemas del aprendizaje y conciencia comunitaria. 
Servicio comunitario del estudiante en el contexto de la educación superior venezolana Ferrer, Juliana y Clemenza, Caterina

- En el área de Ciencias políticas los estudiantes llevarán a cabo el servicio comunitario prestando Asesoría Jurídica en las Alcaldías.

- En el área de Ingeniería, los estudiantes realizarán obras comunitarias relacionadas con la comunidad y según la carrera a la que pertenezcan.

\section{Universidad Dr. Rafael Belloso Chacín (URBE)}

- Se creo la Dirección de Responsabilidad Social Universitaria, la cual tiene como objetivo principal organizar la estructura académica y logística de la labor del servicio comunitario estudiantil, sensibilizando la actuación de la Universidad ante las necesidades de su entorno y dando respuestas concretas.

- El estudiante podrá llevar a cabo el servicio comunitario en el municipio o comunidad donde vive, siempre y cuando exista un proyecto/programa en el mismo y además la corresponsabilidad (acuerdos), entre la comunidad y la universidad.

- Se conformarán equipos de alumnos por carrera o de diversas áreas para llevar a cabo el servicio comunitario, esto dependerá de la necesidad del proyecto/programa.

- Resulta importante destacar el trabajo realizado por esta universidad en cuanto a trabajo comunitario se refiere evidenciando una participación con resultados por las comunidades de manera escrita, al punto que para el período septiembre-diciembre 2006, se atendieron un total de cincuenta y dos (52) comunidades para un total de ciento treinta y dos (132) proyectos y la participación de mil setecientos seis (1706) estudiantes, siendo ésta la primera convocatoria.

\section{Reflexiones Finales}

El compromiso social, de la labor educativa debe guiar la acción, con el fin de transformar las instituciones y comunidades verdaderamente democráticas, para lo cual éstas deben estar inmersas en su contexto inmediato y ser actores para el cambio desde una perspectiva de ciudadanía social, vinculada necesariamente a una perspectiva participativa inclusiva, como epicentro de una construcción de la convivencia comunitaria.

En definitiva, la colaboración activa de las instituciones universitarias, no es más que una preocupación ética para una educación de calidad, que exige el desarrollo de los valores desde la convivencia con las comunidades, como manera más coherente de darle significado al concepto de responsabilidad universitaria, bajo una cooperación que permita la armonización y cualificación de los contextos donde ésta se lleva a cabo. Este debe ser el fin último de una educación que se defina como democrática, siendo necesario que todas y todos se planteen y den respuesta a las demandas comunitarias. 
De acuerdo con lo anterior, el éxito de las políticas de participación y poder populary particularmente la Ley de Servicio Comunitario para el estudiante de Educación Superior, depende de la posibilidad identidad colectiva, la cual permite la movilización de los miembros de la comunidad en búsqueda de soluciones conjuntas con la comunidad universitaria; así como tener una visión del contexto y dinámica globales para comprender la profundidad de esta Ley en su relación con las comunidades afectadas, que refuercen la participación comunitaria y el poder popular, en términos de un proceso educativo comunitario, estableciendo algunos ejes de comprensión a fin de que éstos puedan interactuar de manera permanente, como forma de acumular experiencias transformadoras y participar de manera corresponsable en la dinámica social venezolana, por la construcción de ciudadanía social de futuro.

.En última instancia se busca llegar al momento en Ley de Servicio Comunitario para el estudiante de Educación Superior, quede establecida como cultura, es decir, como fundamento de las formas de relación, producción, creación, y reproducción de la sociedad, establecida a todo nivel al ser interiorizada por individuos y actores sociales como una manera natural de vinculación entre la Universidad y las comunidades.

\section{Referencias Bibliográficas}

Barylko, Jaime (2005). Reflexiones filosóficas. En la búsqueda de la verdad. Editorial Ariel. Argentina.

Bronfman, Mario; Gleizer, Marcela (1994). Participación comunitaria: necesidad, excusa o estrategia? O de qué hablamos cuando hablamos de participación comunitaria. Cad. Saúde Pública vol. 10 n. 1 Río de Janeiro. pp. 109-110.

Cortina, Adela; Castiñeira, Ángel; Conill, Jesús; Domingo, Agustín; García, Domingo; Lozano, José Félix y Lozano, Joseph María (1997). Rentabilidad de la ética para la empresa. Primera Edición. Fundación Argentaria -Visor, C.A. Madrid. pp. 141-152.

De la Riva, Francisco (1993). Investigación participativa y auto formación grupal. En documentación social. Madrid. pp. 141-153.

De Roux, G.; Pedersen, D.; Pons, P.; Pracilio, H. (1990). Participación social y sistemas locales de salud. In: Los Sistemas Locales de Salud (OPS, org.), pp. 28-49, Washington: OPS.

Etkin, Jorge (1996). La empresa competitiva. Grandeza y decadencia. Un cambio hacia una organización viable. Mc Graw Hill. Chile. En: FERRER SOTO, Juliana. (2006). La Corresponsabilidad como principio rector para el Servicio Comunitario desde las Universidades Venezolanas. Trabajo Final presentado en el Postdoctorado de Gerencia de las Organizaciones. Universidad Rafael Belloso Chacín. p. 12.

Ferrer, Juliana (2006). La Corresponsabilidad como principio rector para el Servicio Comunitario desde las Universidades Venezolanas. Trabajo Final presentado en el Postdoctorado de Gerencia de las Organizaciones. Universidad Rafael Belloso Chacín. pp. 11-50.

Figueroa, Luis (2006). Formación Docente: Complejidad y profesionalización. En Revista ETHOS Educativos. No.35. enero-abril. México.

Gwatkin, D.; Wilcox, J. y Wray, J., (1979). Can Interventions Make a Difference? The Policy Implications of Field Experi- 
Servicio comunitario del estudiante en el contexto de la educación superior venezolana Ferrer, Juliana y Clemenza, Caterina

ment Experience. Report to the Word Bank, Washington D.C.: World Bank. Editorial Mimeo. pp. 45-85.

Kliksberg, Bernardo (2000). Seis tesis no convencionales sobre participación. Capital Social y Cultura: Claves Estratégicas para el Desarrollo. Banco Interamericano de Desarrollo. Fundación de Cultura Económica.

Llano Carlos (1997). Dilemas éticos de la empresa contemporánea. Fondo de Cultura Económica. México.

Lozano, Josep (1999). Ética y Empresa. Editorial Trotta. México.

Manderson, L.; Valencia, L.; Thomas, B. (1991). Bringing the people. In: Community Participation and the Control of Tropical Diseases. Resource Papers for Social and Economic Research in Tropical Diseases № 1, UNDP/WORLD BANK/WHO/TDR, Geneve: World Health Organization. pp. 34-42.

Morín, Edgar (1995). Introducción al pensamiento complejo. Barcelona.

Morín, Edgar (1999). Los siete saberes necesarios para la educación del futuro. UNESCO- París.

Asamblea Nacional. República Bolivariana de Venezuela (2006). La Ley Orgánica de Participación Ciudadana y Poder
Popular, aprobada en primera discusión el 22 de Noviembre de 2001 y el 19 de Julio de 2006 (Segunda Discusión).

Asamblea Nacional. República Bolivariana de Venezuela (2006). Ley de Servicio Comunitario del estudiante de Educación Superior. Gaceta Oficial 38.272 de Fecha 14 de septiembre de 2005.

República Bolivariana de Venezuela (2001). Plan Nacional de Desarrollo Económico y Social de la Nación 2001-2007. Caracas, (Venezuela).

República Bolivariana de Venezuela (2006). Ministerio de Educación Superior. Misiones Educativas. Documentos mimeografiados. Caracas (Venezuela).

Romero, Bievenida; Sarmiento, Marcos; Abreu, Mercedes (2006). Cómo diseñar proyectos comunitarios. Fundacite. 3era edición. Venezuela.

Winch, P.; Kendall, C.; Gubler, D. (1991). Community Participation in the Control of vector-Borne Diseases: A Review of Selected Activities in Latin America. Trabalho apresentado no Simpósio "Community Participation and Control Efforts in Developing Countries", Annual Meeting of the American Mosquito Control Association, Louisiana. 